\title{
HELP-WSN-A Novel Adaptive Multi-Tier Hybrid Intelligent Framework for QoS Aware WSN-IoT Networks
}

\author{
J. Sampathkumar* and N. Malmurugan \\ Mahendra College of Engineering, Salem, 636106, India \\ *Corresponding Author: J. Sampathkumar. Email: sampathkumar1980@gmail.com \\ Received: 09 May 2021; Accepted: 01 September 2021
}

\begin{abstract}
Wireless Sensor Network is considered as the intermediate layer in the paradigm of Internet of things (IoT) and its effectiveness depends on the mode of deployment without sacrificing the performance and energy efficiency. WSN provides ubiquitous access to location, the status of different entities of the environment and data acquisition for long term IoT monitoring. Achieving the high performance of the WSN-IoT network remains to be a real challenge since the deployment of these networks in the large area consumes more power which in turn degrades the performance of the networks. So, developing the robust and QoS (quality of services) aware energy-efficient routing protocol for WSN assisted IoT devices needs its brighter light of research to enhance the network lifetime. This paper proposed a Hybrid Energy Efficient Learning Protocol (HELP). The proposed protocol leverages the multi-tier adaptive framework to minimize energy consumption. HELP works in a two-tier mechanism in which it integrates the powerful Extreme Learning Machines for clustering framework and employs the zonal based optimization technique which works on hybrid Whale-dragonfly algorithms to achieve high QoS parameters. The proposed framework uses the sub-area division algorithm to divide the network area into different zones. Extreme learning machines (ELM) which are employed in this framework categories the Zone's Cluster Head (ZCH) based on distance and energy. After categorizing the zone's cluster head, the optimal routing path for an energy-efficient data transfer will be selected based on the new hybrid whale-swarm algorithms. The extensive simulations were carried out using OMNET++-Python userdefined plugins by injecting the dynamic mobility models in networks to make it a more realistic environment. Furthermore, the effectiveness of the proposed HELP is examined against the existing protocols such as LEACH, M-LEACH, SEP, EACRP and SEEP and results show the proposed framework has outperformed other techniques in terms of QoS parameters such as network lifetime, energy, latency.
\end{abstract}

Keywords: Internet of things; extreme learning machines; zones' cluster head; hybrid whale-swarm 


\section{Introduction}

Over the last few years, WSN assisted Internet of Things paradigm [1] has evolved as one of the biggest technological advances in the modern era of science. WSN assisted IoT networks to consist of sensor nodes, actuators, transceivers and many more devices for better data collection, monitoring and controlling. These hubs presented in WSN supported IoT networks create the physical objects aware of different attributes in the deployed networks and trigger an event in coordination with the other nodes in the network. Also, these hubs are usually energy consuming which demands intelligent techniques to increase network lifetime [2]. To countermeasure, these concerns, different intelligent algorithms were where the clustering algorithms occupy the major share for a WSN-IoT Implementation. The preliminary moto of the routing protocols based on clustering is to choose the outstanding energy-efficient Cluster Head $(\mathrm{CH})$ to route the other nodes via in it. This $\mathrm{CH}$ has accountability for the data collection and aggregation which then transmit the aggregated data to BS. To countermeasure all the above-mentioned issues, this paper proposes the new protocol HELP-DAR (Hybrid Efficient Learning Protocol using Dynamic Adaptive Routing), a hybrid multi-tier and intelligent energy-efficient routing protocol which integrates the powerful extreme learning machines for the selection of zone-based $\mathrm{CHs}$ along with the hybrid metaheuristic algorithm for an efficient and optimal zonal routing protocol. The main contribution of the paper is as follows as

(1) A multi-tier hierarchical framework for WSN-assisted IoT networks in a Smart Health care system has been proposed. The proposed framework aims to transmit the data in an energy-efficient network with less delay and high performance.

(2) Usage of Single Feed Forward learning models in the Cluster Head election has been introduced with the adaptive energy-distance data training vectors.

(3) Integration of hybrid whale optimized swarm routing algorithms to achieve lossless data transmission and to prolong the network lifetime.

The paper flow is presented as follows: The related works regarding the different routing protocols for WSN and WSN assisted networks has been discussed in Section-II. Section-III discusses the network model, energy models and proposed frameworks with an effective selection of Cluster heads with effective implementation of the hybrid routing algorithm. The experimental setup, simulation parameters, result analysis were discussed in Section-IV. The Conclusion is presented in Section-V.

\section{Related Works}

Jaiswal et al. (2020) designed a novel routing protocol for WSN. The proposed model is energy efficient and guarantees the improvement of QoS. The routing protocol is designed to withstand high traffic loads. The path selection process is carried out based on the lifetime of the link, reliability and traffic intensity at the surrounding nodes. The result shows better energy saving and better packet delivery ratio (PDR). The limitation of the model is moderate consideration of delay in the intermediate layers, which can further increase the delay in the overall network [3].

Sambo et al. (2019) performed an elaborate study on clustering in large WSN models. Hierarchy issue arises while clustering the nodes in the WSN's. Hence, intelligent clustering schemes are needed to optimize the WSN systems. In the study, based on 10 different parameters in WSN, the clustering algorithm are categorized to study the overall efficiency of various protocols. The study didn't consider the spectrum efficiency of WSN's while clustering [4]. 
Zhao et al. (2018) developed a CH selection protocol that relies on LEACH. The ZigBee modules are employed to calculate the residual energy and IP of the node to update the cluster head selection equation. This type of modification drastically enhances energy efficiency [5].

Sharma et al. (2018) proposed an enhanced mobility aware energy-efficient routing protocol for IoT (EMAEER) to optimize the routes to establish point-to-point communication. The mobile nodes are always held within the network to further reduce the loss of energy. The simulation is compared with RPL, P2R RPL and ER-RPL algorithms and the energy consumption is reduced at a rate of $9.61 \%$ providing better packet delivery. The model withholds only a minimum number of mobile nodes [6].

Neetesh et al. (2019) put forth an idea of energy conservation in WSN as a green routing protocol that enables a fork and uses modified PSO (Particle Swarm Optimization) to expand the network's life competency. The system outperforms even in high collision IoT WSN's. Intelligent hardware deployment is quite challenging in this model [7].

Dwi Widode et al. (2019) developed a novel cluster head selection model using the reinforcement learning method. RC being an MC based scheme learns the behaviour of clusters in the entire network. The sorting of clusters is carried out from the pre-learned ideas and then transmission of packets is attained. The method enhances life and reduces the energy usage in the WSN's. The model lagged to transmit data due to the prolonged collision of nodes [8].

Chriki et al. (2019) proposed an idea of positioning the clusters by using multiple class SVM structures and with the received signal strength measurements. The model is tested with real datasets being collected from a hospital and a laboratory area. The method uses a neural network architecture to perform efficient routing. The false transmission rate is reduced up to $90 \%$ in the proposed scheme. The model is suitable only for small-scale network applications [9].

Behera et al. (2017) developed an improved model of stable Election Routing Protocol. This model uses a threshold value to decide the cluster head in the network. This aids in the optimal decision of cluster heads. The efficiency is improved up to $56 \%$ than state-of-art models [10].

Li et al. (2019) introduced a deep learning-based approach with bat optimization to training the model. The extreme learning approach decides the path using a threshold onset and the bioinspired Bat optimizer decides the optimal solution. The node decaying is the main issue faced in this optimization scheme. When a node decays, the injection of a new node needs to be considered for effective communication [11].

Anurag et al. (2018) developed a "scalable and Energy Efficient Routing Protocol" to conserve energy in WSN systems. The methods divide the network based on the distance and congestion, clustering is performed. The stability of the model is quite challenging in large area WSN's [12].

\section{Proposed Framework}

\subsection{System Overview}

Fig. 1 represents the HELP Multi-Tier Clustering framework for the WSN assisted IoT networks. The nodes belong to this framework are static and dynamic in nature which relies on the requirements of the application. In this proposed framework, rectangular shape networks area is implemented for the processing as mentioned in [12]. The cloud server will act as a BS layer, which occupies the top position in the hybrid framework. Based on distance (low, medium, long) the total network area is subdivided into different zones every category of zones has sub-zones and it is denoted as Cluster. As the distance increases, the increased number of cluster heads 
leads each sub-zones. The Low distance and Medium distance zones have a minimum area with a minimum No. of $\mathrm{CHs}$ whereas long-distance zones have a maximum area with a maximum No. of CHs. The upper zones not only aggregate the data from the other zones but also depends on the traffic in the preceding zones. The intracluster communication is reduced by increasing the number of $\mathrm{CHs}$ which depends upon area and distance.

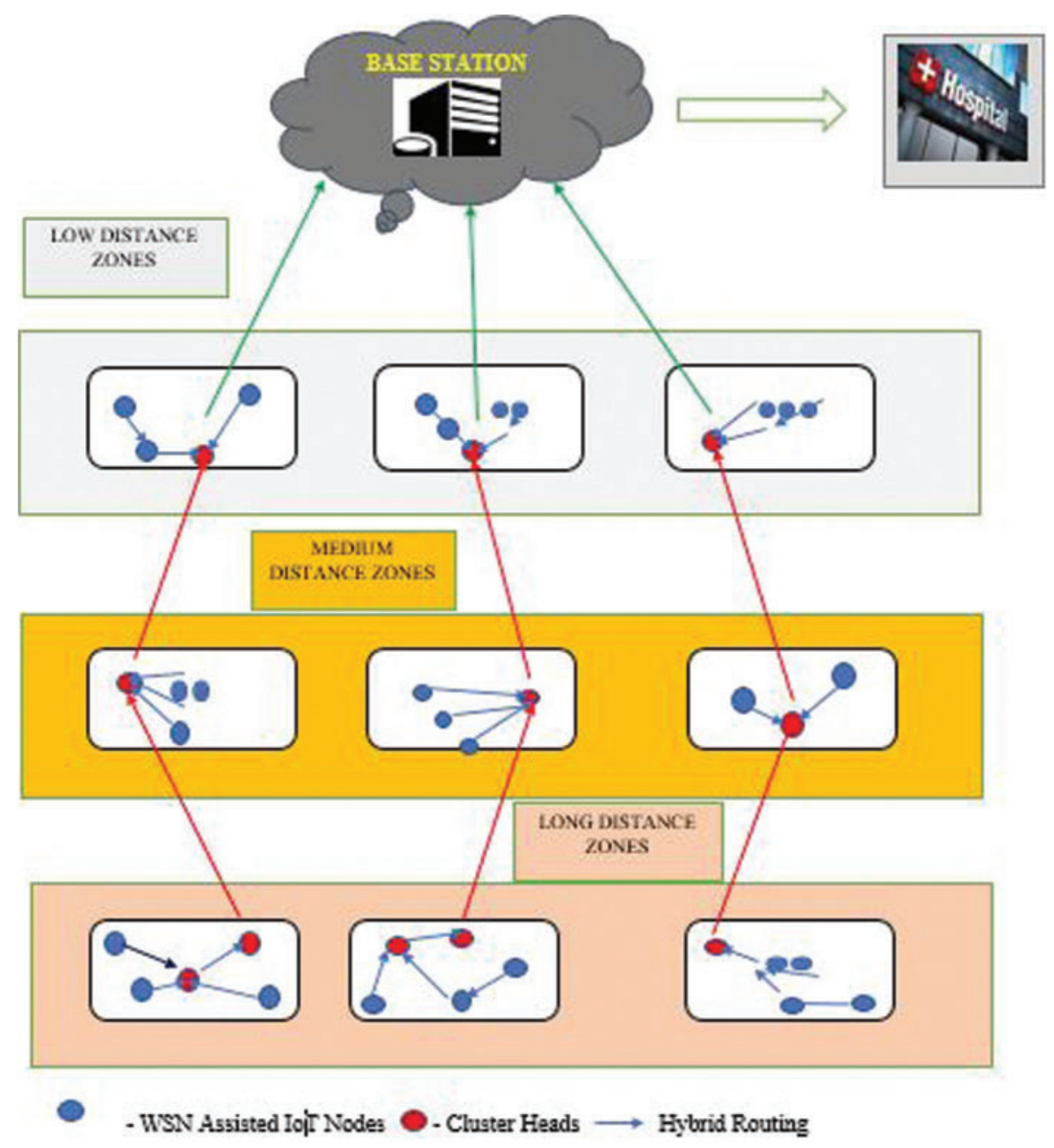

Figure 1: Hybrid intelligent framework (multi-tier clustering framework)

In this research, smart healthcare systems are taken for investigation. In this framework, to monitor and collect body parameters, humans are well equipped with IoT wearable gadgets. Various Zonal $\mathrm{CHs}(\mathrm{ZCH})$ are incorporated to transmit the health care data Cloud $\mathrm{BS}$ (CBS). This is considered to be a continuous process until CBS receives all the health care data from the upper zone $\mathrm{ZCH}$. Efficient inter-zonal communication and intra-cluster communication are effectively achieved by the hybrid optimization algorithm which will be discussed in the preceding section. As the nodes detect the critical health data from the patient, an alert message is evoked from the particular node and it is transmitted to the CBS so the CBS broadcast the emergency message to all nearby hospitals with the ZCH's information along with patients Id so that 
ambulance can be sent from the hospitals to reach the destination place with minimum time. Since wearable devices are mounted on a patient's body, frequent recharging and replacement of batteries are quite complex, hence intelligent and effective communication is required to avoid the fatal ends.

\subsection{Network Model}

A Proposed framework consists of directed acrylic graph $G=(x, y)$. Where $x \rightarrow$ the vertex of the hubs which integrates $\mathrm{ZCH}$ and other monitoring hubs, these hubs are distributed randomly within the health care systems. The following characteristics of the proposed framework are listed

(1) Nodes in the network are considered wearable IoT devices, which are used to sense clinical parameters such as temperature, breath rate and pulse rates.

(2) To complete the task, various programs were embedded to sense the programs and unique identities are given to each node for better identification. (ID)

(3) Each $\mathrm{ZCH}$, node is considered as both static and dynamic which are then typically required for WSN applications.

(4) The proposed framework is dedicated to the smart health care environment, which senses the clinical data from the patients.

(5) Initial Energy has been fairly distributed in the networks and purely location-unaware as well as not equipped with GPS.

(6) Among all the nodes Communication links are truly symmetric. Therefore, every node can do the distance estimation based on the received signal strength.

\subsection{Energy Models}

The paper uses the energy model from [13-15]. Data handling in WSN links consume energy in three different phases namely, data transmission mode, reception phase and aggregation phase. The energy being consumed in the data transmission phase be $E_{T X}$. The energy consumed in the reception phase be $E_{R X}$ and the energy consumed in the aggregation phase be $E_{D A}$. The energy being utilized in the aggregation $E_{D A}$ remains fixed for the entire cycle, whereas the energies $E_{T X}$ and $E_{R X}$ will vary based on the situation. Let $d$ be the distance between the transmitting sensor nodes to the receiver head. The energy value $E_{T X}$ always depends on the distance $d$. Let $d_{0}$ be the threshold value to develop a multi-state model and if $d<d_{o}$

$E_{T X}= \begin{cases}l \times E_{\text {elec }}+l \times \epsilon_{f s} \times d^{2}, & \text { if } d<d_{0}, \\ l \times E_{\text {elec }}+l \times \epsilon_{m p} \times d^{4}, & \text { otherwise }\end{cases}$

where $E_{\text {elec }}$ is the electric energy needed to convert single bit data into a signal, $l$ is the length of the data and $\epsilon_{f s}$ and $\epsilon_{m s}$ are power consumption for the multi-state model to satisfy the threshold $d_{0}$,

$d_{0}=\sqrt{\frac{\epsilon_{f s}}{\epsilon_{m p}}}$

The energy utilized at the reception phase converts the received signal into data and $E_{\text {elec }}$ is the electric energy consumed for this conversion. The relationship between based on length of the received data $l$ be

$E_{R X}=l \times E_{\text {elec }}$ 


\subsection{Working Mechanism}

The proposed HELP framework works on the following phases such as Initialization Phase, Cluster Head Formation, Data Routing Mechanism and Aggregations. The detailed description of the proposed model is discussed in the following section'

\subsubsection{Initialization Phase}

In this phase, sensor nodes are deployed in a particular area in the form of a rectangular shape by following the area-subdivision algorithm as mentioned by Anurag et al. [12] Once the sensor nodes are placed in the different zonal areas, CBS sends the beacon messages to all nodes present in the different zones. Each node transmits the data to CBS in the following format such as Zone ID, Node ID, Energy ID and distance d. Once the data frames are received by the CBS, CBS extracts the different features from the above data frame format, CBS adopts the Extreme Learning Machines for further processing.

\subsubsection{Cluster Head Decision}

Once the initialization phase is established, $\mathrm{ZCH}$ selection will be evoked by the base station, which runs the proposed extreme learning machines (ELM) on it. Initially, the CBS is trained with the data models with the following criteria

(1) The ZCH with high residual energy can keep support uniform energy depletion in the networks.

(2) The nodes which are nearer to the BS can help to prolong the networks lifetime by significantly decreasing the communication distance

The two features are used mainly to train the CBS to select the $\mathrm{ZCH}$ in the network scenario. For the distance calculation, the model uses the Euclidian distance (ED) and average distance (AD) between the CBS and nodes. Subsequently, the value of the energy in the nodes and distance is used as the $\mathrm{ZCH}$ selection. In this case, the adaptive energy-distance parameter is calculated in which energy consumption per average distance will be measured as a threshold. The mathematical expression for calculating the energy and distance vectors are calculated as mentioned in [16]. The working mechanism for the Extreme Learning Machines for selecting the Cluster head is given as follows

\subsection{Extreme Learning Machines - an Overview}

The $\mathrm{CH}$ is selected based on the ELM proposed by Huang et al. [16]. These ELM network main features are high speed and accuracy. This network has universal function approximation capabilities and a single HL (hidden layer). The main advantage of this ELM framework is the HL needs no to be tuned necessarily. Here, HL needs to work with activation function (i.e., sigmoid function) which is vastly differentiable. ELM, the system yield is expressed as follows (4)

$f_{L}(x)=h(x) \beta=h(x) H^{T}\left(\frac{1}{C} H H^{T}\right)^{-1} O$

where $\mathrm{x} \rightarrow$ input, $\beta \rightarrow$ "Output weight vector", $\mathrm{H}(\mathrm{x}) \rightarrow$ output $\mathrm{HL}, \mathrm{H} * \rightarrow$ inverse of $\mathrm{H}$

The kernel function is incorporated for better accuracy in ELM. In the proposed framework, ELM is used to select the CHs based on the Energy \& distances. The two or more CHs are selected based on the above features to ensure the increased lifetime in the network. The ELM 
is trained with a sigmoidal activation function with a learning rate of 0.001 . The pseudo-code of the proposed ELM for the selection of Cluster head is given as follows

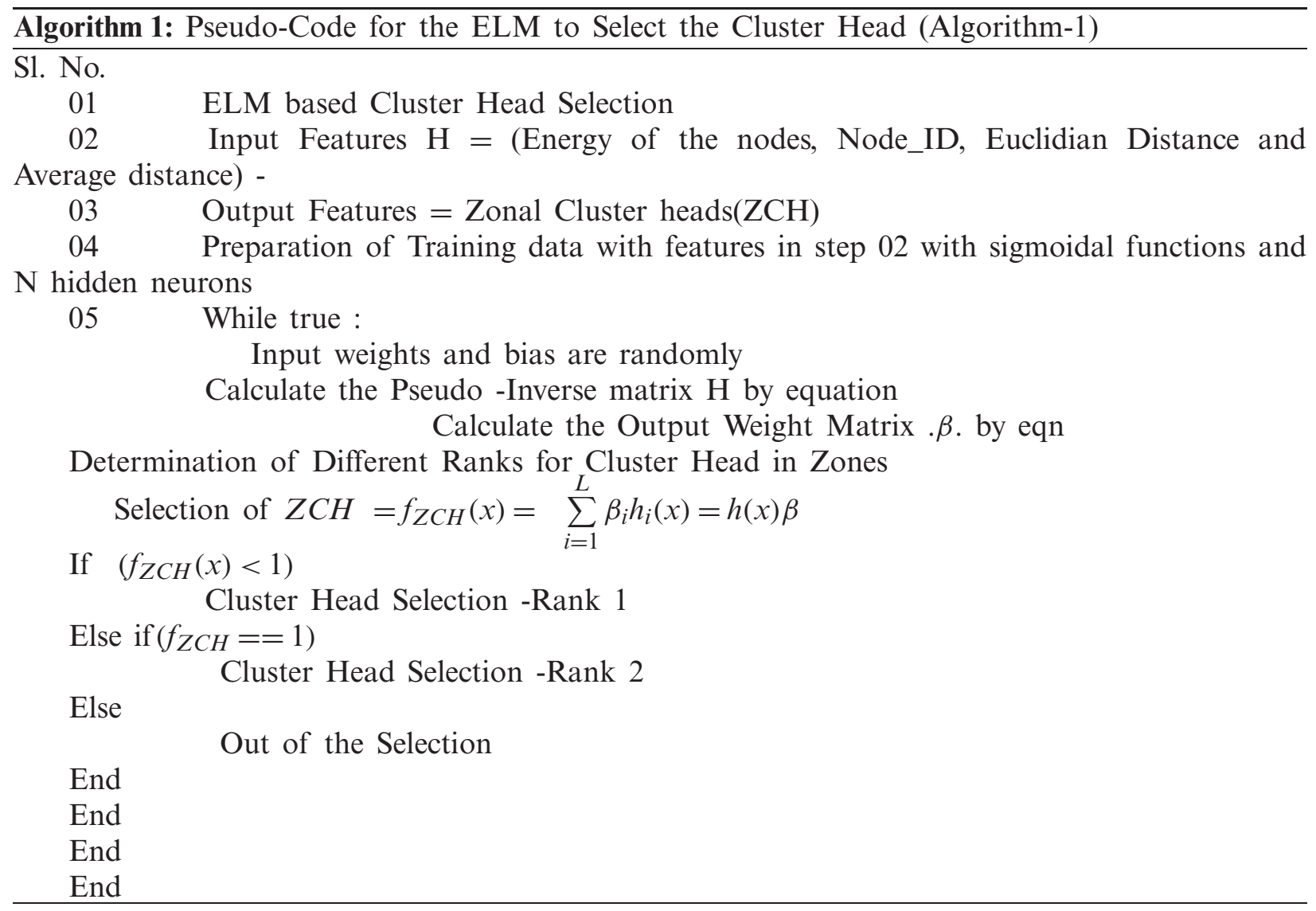

\subsubsection{Routing Phase}

After the $\mathrm{ZCH}$ selection in every zone by adopting algorithm-1, routing will take place. It can be noticed from the above energy model that energy consumption is proportional to the communication distance. Hence optimized routing path has to be selected for an increased lifetime of networks. To save this purpose, a hybrid whale-firefly algorithm is used for selecting the optimal path. The working mechanism utilizes the firefly [17] and whale algorithm [18]

\subsection{Proposed Optimizer for Routing}

The proposed protocol uses the hybrid combination of the firefly [17] and whale algorithm [18] for finding the optimized energy-efficient path. Since the WSN-assisted IoT networks cover a larger area, usage of metaheuristic algorithms may lead to the trapping problem which may lead to low efficiency. Hence the proposed protocol uses the hybrid combination of firefly and whale algorithms for finding the optimized energy-efficient path. These hybrid combinations truly reduce the trapping problem and find its suitability for the larger area coverage. For the best performance, a multi-objective fitness function has been designed

$F(E)=c 1|N(C H i)|+c 2 * \sum(N E)+c 3 * \sum(N D)$ 
where c1, c2, c3 are randomly assigned (randomly assigned between 0 to 1), $\mathrm{N}(\mathrm{CHi}$ ) is the complete neighbour list of nodes in particular zones, NE is the residual energy of every node, and ND represents the distance between the neighbours in the zones. To obtain the energy-efficient path with lossless data transmission, iteration is fixed to maximum numbers and the fitness value of the proposed routing is computed at every iteration. The proposed working mechanism's complete pseudo-code is given as follows

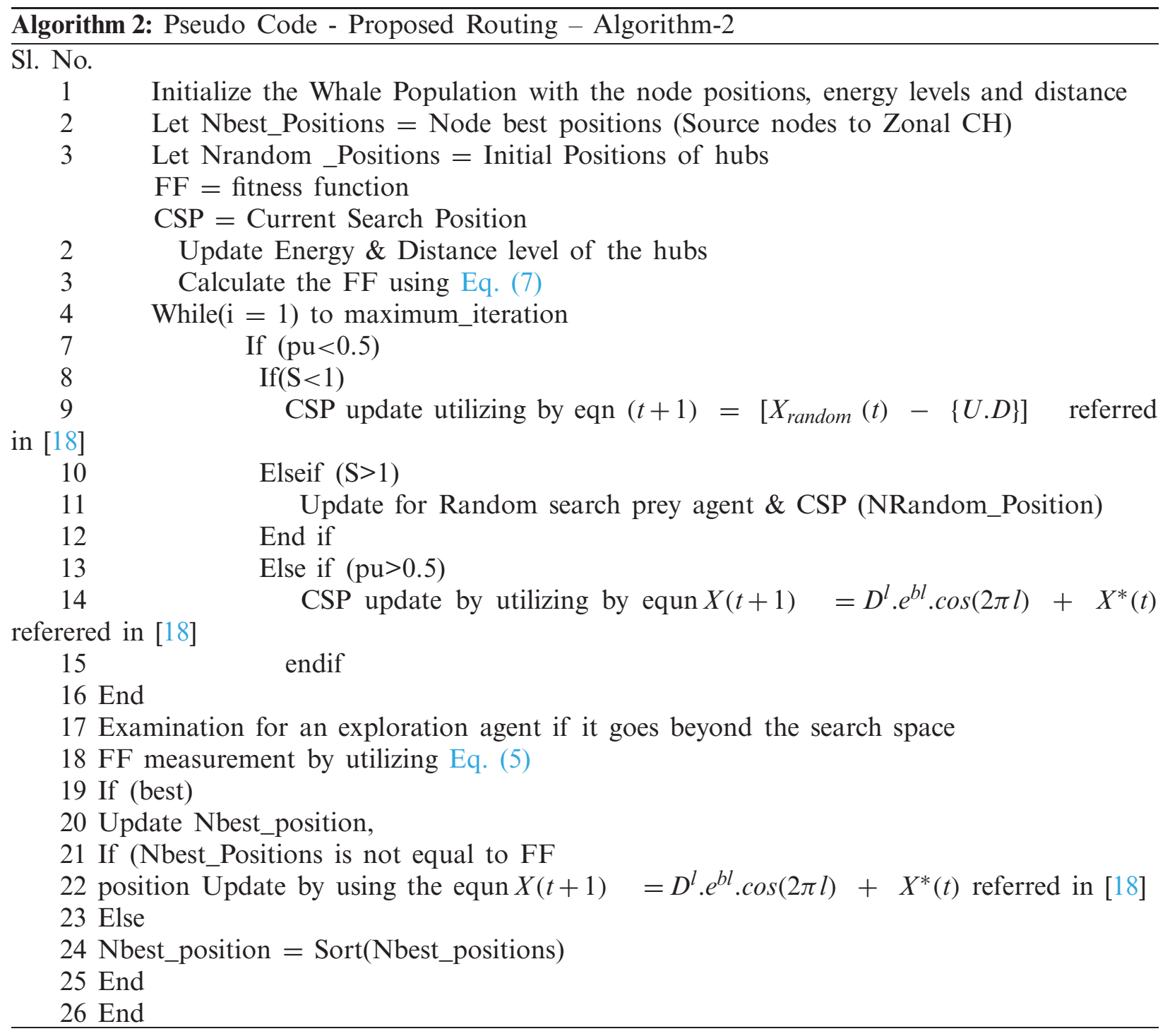

\subsection{Aggregation Model}

In this framework, eligible $\mathrm{CHs}$ performs the data aggregation process, which decreases the energy depletion of the hubs. the data aggregation model adopted in the proposed framework and through cluster heads data, CBS receives the data from all the nodes. 


\section{Experimental Setup}

We have validated the performance of the proposed protocol in two ways such as algorithm centric evaluation and performance-centric validation respectively. Since the proposed protocol has integrated the Extreme Learning Machines for Cluster Head Selection, performance metrics such as accuracy, precision and recall have been calculated utilizing the following mathematical formula

Accuracy $=\frac{\mathrm{DR}}{\mathrm{TNI}} \times 100$

Sensitivity $=\frac{\mathrm{TP}}{\mathrm{TP}+\mathrm{TN}} \times 100$

Specificity $=\frac{\mathrm{TN}}{\mathrm{TP}+\mathrm{TN}} \times 100$

where DR $\rightarrow$ Detected Results, TNI $\rightarrow$ Total number of Iterations, TP $\rightarrow$ True Positive, TN $\rightarrow$ True Negative

True positive values are the number of $\mathrm{CHs}$ selected based on the residual energy, distance and channel properties. True negative is the number of non-cluster heads nodes when they indeed to be normal. Nearly 3300 data were collected in wireless assisted IoT scenario using OMNET ++ Python API scenario and the proposed learning algorithm has been implemented with PythonTensor Flow in which the $80 \%$ were used for training and $20 \%$ were used for Testing. Tab. 1 presents the proposed protocol parameter for the performance validation. Furthermore, to evaluate network lifetime and energy comparison, we have used First Node Dead Metrics (FNDM), Half Node Dead Metrics (HNDM), Last Node Dead Metrics (LNDM) and No. of dead nodes after each round. The number of nodes received by the sink (base station) through adaptive hybrid protocol is denoted as one round. FNDM, HNDM, LNDM represents the duration between the rounds, which illustrates the point of time when the first nodes, half nodes and last nodes become dead [12]. The complete simulation scenario for the proposed HELP protocol is shown in Fig. 4. OMNET++ has been used for the complete implementation of the proposed protocol. Tab. 1 presents the complete simulation parameters utilized for the experimentation.

\subsection{Results and Discussion}

The proposed HELP protocol performance is in a two-tier fashion, which is discussed in this section. In the first tier, performance metrics of proposed Extreme Learning Machines (ELM) has been evaluated and compared with the other existing algorithms such as Reinforcement Learning Algorithms (RL) [8], "Support Vector machines" (SVM) [9], Artificial Neural Networks (ANN) [19], Naïve Bayes Algorithms (NB) [20], K-Nearest Neighborhood algorithm (KNN) [21-23].

\section{Algorithm Centric Evaluation}

The proposed model mentioned were trained from the different energy, distance vectors using sigmoid activation function with the learning rate of $1 \times 10^{-3}$ learning and moving average decay of 0.9999. Furthermore, the performance of the proposed ELM in terms of training \& testing accuracy was calculated for the different number of input neurons which is shown in Fig. 2 
Table 1: Simulation parameters utilized for the experimentation

\begin{tabular}{lll}
\hline Sl.no & Simulation parameters & Description \\
\hline 1 & Initial energy & $5 \mathrm{~mJ}$ \\
2 & Energy distribution to all nodes & $50 \mathrm{~nJ} / \mathrm{bit}$ \\
3 & Energy threshold & $30 \mathrm{~nJ} / \mathrm{bit}$ \\
4 & Distance threshold & $2-5 \mathrm{~m}$ \\
5 & Number of bytes transmitted & $6400 \mathrm{bites}$ \\
6 & Topology used & Static Nodes \\
7 & No of neurons used for training the CBS & 200 \\
8 & Learning rate & 0.001 \\
9 & ZCH selection probability & $1(10 \mathrm{nodes})$ \\
10 & Energy of aggregation & $5 \mathrm{~nJ} / \mathrm{bit}$ \\
11 & Number of nodes employed & 100 \\
12 & Area of coverage & Dynamic \\
\hline
\end{tabular}

From Fig. 2, it is clear that the accuracy of the proposed algorithm in determining the zonal cluster heads is found to be $97.25 \%$ at 75 hidden neurons. Even though accuracy has reached its maximum at 75 hidden neurons, we have taken the optimal value of 100 neurons for training and testing the different data vectors.

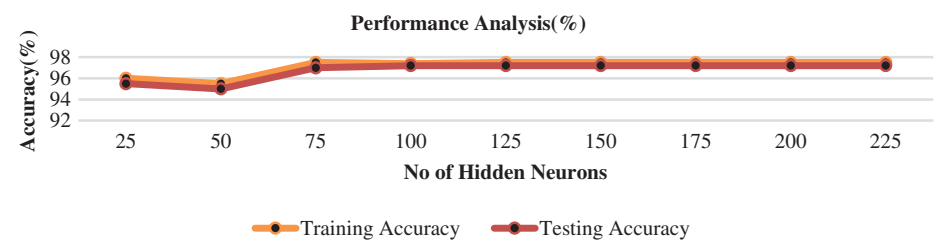

Figure 2: Performance evaluation of the proposed extreme learning machines for different neurons

Tab. 2 shows the comparative analysis of performance metrics for the different learning models for the various area coverage. In all scenarios, the accuracy, precision and recall are found to be $97.25 \%, 96.5 \%$ and $96.25 \%$ respectively. Also, the accuracy of the proposed algorithm has exhibited a 5\% increase than KNN, 7.25\% increase than Naïve Bayes (NB), 8\% increase than Artificial Neural Networks(ANN), and 5.25\% increase than Reinforcement Learning(RL) respectively. Also, tables clearly illustrate that the proposed learning model outperforms the other existing learning models and proves its suitability in the detection of the different zonal cluster heads.

\subsection{Network Centric Evaluation}

In this section, QoS metrics such as network lifetime, energy analysis, PDR (packet delivery ratio) were calculated for the proposed model under increasing network size and compared with the other existing static and dynamic models. 
Table 2: Comparative analysis of performance for different learning models@ different area

\begin{tabular}{lllll}
\hline Sl.no & Algorithm details & \multicolumn{3}{l}{ Performance metrics $(\%)$} \\
\cline { 3 - 5 } & & Accuracy (\%) & Recall (\%) & Precision (\%) \\
\hline 01 & RL [8] & 92.0 & 91.0 & 90.5 \\
02 & SVM [9] & 92.5 & 91.0 & 90.5 \\
03 & ANN [19] & 88.5 & 87.5 & 86.5 \\
04 & NB [20] & 90.5 & 89.0 & 88.5 \\
05 & KNN [21] & 90.5 & 88.5 & 89 \\
06 & Proposed ELM & 97.25 & 96.5 & 96.25 \\
\hline
\end{tabular}

\subsubsection{Network Life Time}

In this analysis, network lifetime has been calculated based on FNDM, HNDM, LNDM statistics under increasing area coverage such as $60 \times 120 \mathrm{~m}, 80 \times 160 \mathrm{~m}, 100 \times 200 \mathrm{~m}$ and $120 \times 240$ $\mathrm{m}$ respectively.

Fig. 3 illustrates the dead node trend of compared protocols. To make it clearer, network lifetime is compared with the FDNM, HDNM and LDNM analysis in the figure.

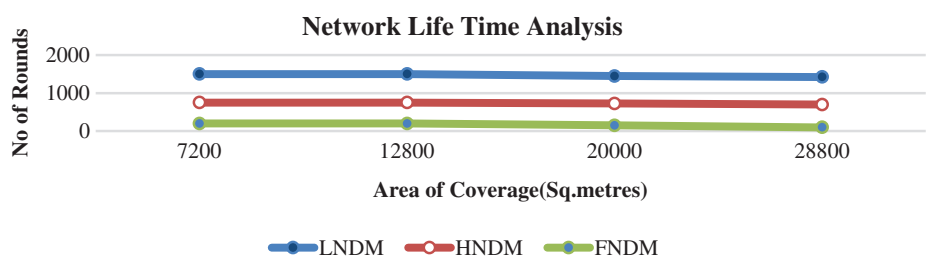

Figure 3: Network life time characteristics for proposed models at different area coverage

Figs. 3 and 4 shows the network lifetime analysis of the proposed HELP model with the changes in the area dimensions. It has been noticed in Fig. 4 when the network size was increased from 7200 Sq.metres to 12000 Sq. meters, the proposed framework remains stable due to the more amount of zones and also the adoption of the optimized routing path to the nodes present in all zones. The large "communication distance" is divided into small multi-hop communications with a large number of $\mathrm{CH}$ selected by the powerful extreme learning machines that distribute the load to the network. The network lifetime of HELP is maintained constant up to network size reaches 20000 square meters and decreases slightly when it exceeds the 20000 square meters whereas other algorithms such M-LEACH, SEP, LEACH decreases exponentially as the area of coverage increases. The other algorithms such as EACRP and SEEP remains almost stable till the network size reaches 12800 sq. Meters but considerably reduces when reaches beyond the 20000 sqm. The proposed HELP performs well and gained a network lifetime even greater than 1500 rounds.

Both SEEP and EACRP divides the coverage area into the number of zones in which SEEP categories more number of zones when compared with the EACRP. But the non-integration of optimization techniques in the SEEP makes the little ineffective in achieving network stability. 
From all the Figures it has been proved that the integration of optimized paths in the zones has enhanced network lifetime.

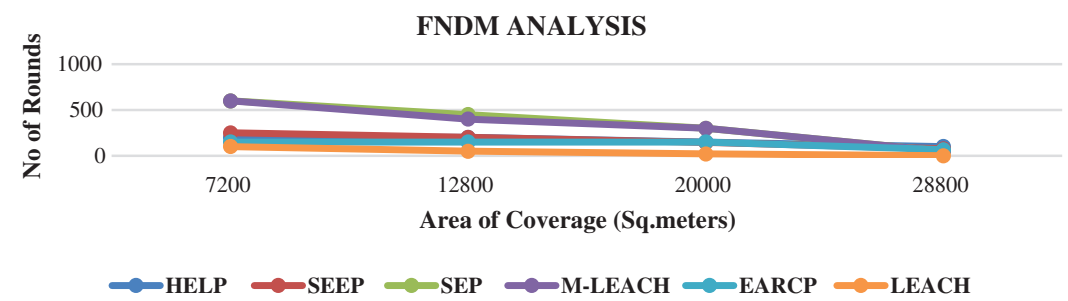

(a)

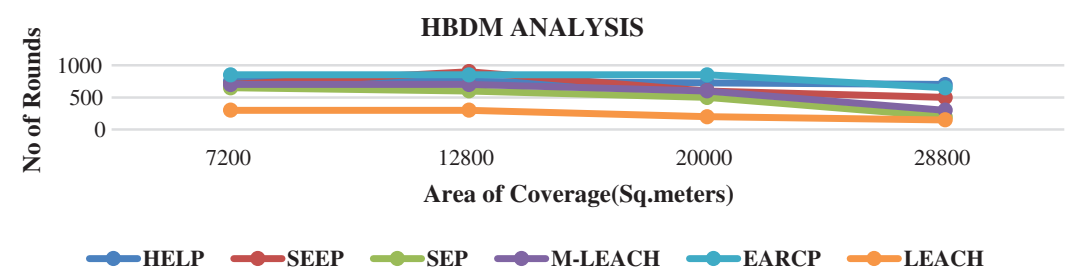

(b)

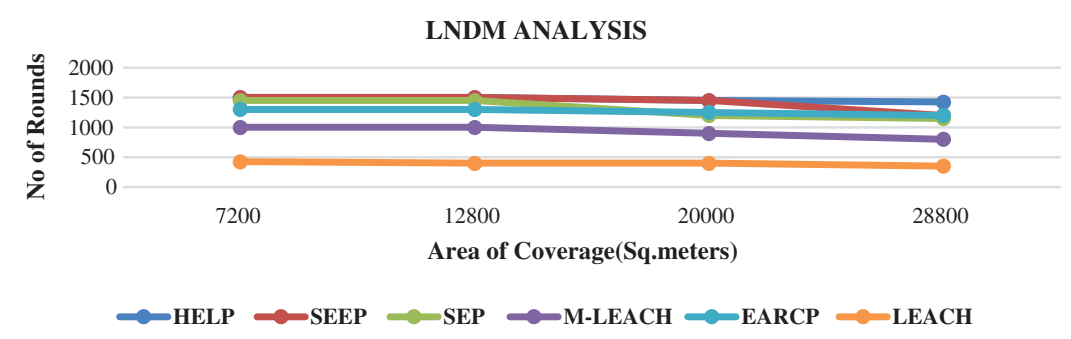

(c)

Figure 4: Network life time analysis at different area coverage (a) FDNM analysis (b) HDNM analysis (c) LDNM analysis

\subsubsection{Energy Consumption}

In this scenario. Total energy depletion is calculated by the below mathematical expression for the increasing area of coverage. To analyze the energy consumption of the networks, 100 nodes were randomly deployed in a different area of coverage which is mentioned section. Total Energy consumption is calculated by using the mathematical expression

Total Energy Consumption: $\sum^{\text {rounds }}\{$ ETtx, rx + EtxCh $\}$

where ETtx, rx is Energy transmitted and Received by the Nodes in the Networks and EtxCh is the transmission and reception energy for the Cluster heads in zones.

Figs. 5 to 8 illustrates the total energy consumption analysis of the compared protocols for all area dimensions from the first to last rounds. Fig. 5 shows the total energy consumption analysis of the different protocols for the area coverage of $60 \times 120 \mathrm{Sq}$. meters. Both LEACH and M-LEACH depletes their full energy as it reaches 600 and 1000 rounds respectively. The other algorithms such as SEP, EARCP, SEEP and Proposed HELP exhibits good performance 
and consumes less energy. Fig. 6 shows the total energy consumption analysis of the different protocols for the area coverage of $8 \times 160$ Sq.meters. In this case, LEACH and M-LEACH algorithms deplete completely at 600 and 400 rounds respectively. The energy consumption of other algorithms such as SEP, EARCP and SEEP exhibits the medium edge of performance whereas the proposed algorithm maintains the same high performance as in the previous case. Fig. 7 shows the total energy consumption analysis of the different protocols for the area coverage of $100 \times 200$ Sq.meters. It is clear, as the area of coverage increases, the performance of LEACH, M_LEACH and SEP degrades gradually even at 300, 600, 1000 rounds respectively. It is also found that the EARCP and SEEP exhibit lesser performance comparatively depletes completely at 1300 and 1500 rounds. Though the area of coverage increases, HELP remains active even greater than 1500 rounds. Fig. 8 shows the total energy consumption analysis of the different protocols for the area coverage of $120 \times 240$ Sq. meters. In this analysis, LEACH, SEP and MLEACH deplete fast as the area increases. Both EACRP and SEEP exhibits good performance in consuming less energy again these algorithms suffers from a faster depletion rate as it drains even at 1200 and 1400 rounds respectively. The plots illustrate that the proposed protocol has a uniform energy depletion curve as contrasted with other WSN-assisted IoT protocols even greater than 1500 rounds. It can be observed from all figures; the proposed HELP exhibits uniformity in energy consumption even though the area of coverage increases. It can be observed, both LEACH, and M-LEACH depletes fast as the area increases. Both EACRP and SEEP exhibits good performance in consuming less energy again these algorithms suffers from a faster depletion rate when the area increases. The plots illustrate that the proposed HELP protocol has a "uniform energy depletion curve" as compared with other existing WSN-assisted IoT protocols even greater than 1500 rounds. The effective selection of areas based on zonal cluster heads interfaced with optimized routing algorithm makes the proposed algorithm consume less energy of $11 \%$ than SEEP, 25\% than EARCP, 34\% than SEP, 75\% than LEACH and M-LEACH respectively.

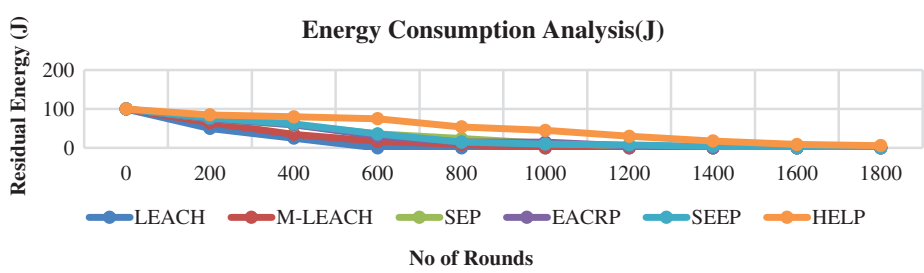

Figure 5: Energy consumption analysis for the different protocols at area of dimension $60 \times 120$ Sq.meters (7200 Sq.meters)

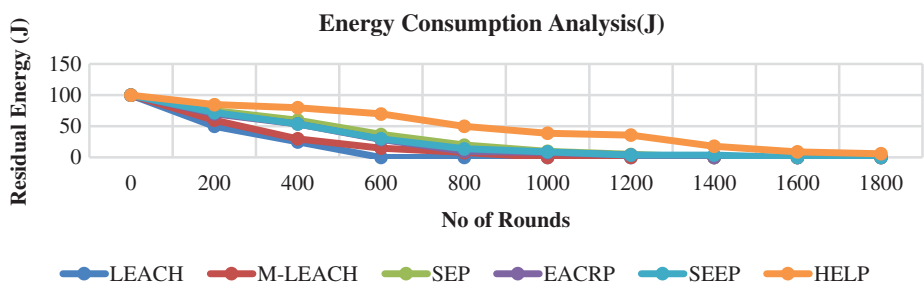

Figure 6: Energy consumption analysis for the different protocols at area of dimension $80 \times 160$ Sq.meters (12800 Sq.meters) 


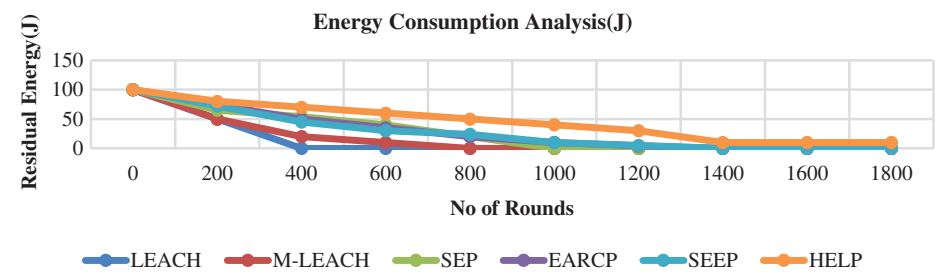

Figure 7: Energy consumption analysis for the different protocols at area of dimension $100 \times 200$ Sq. meters (20000 Sq.meters)

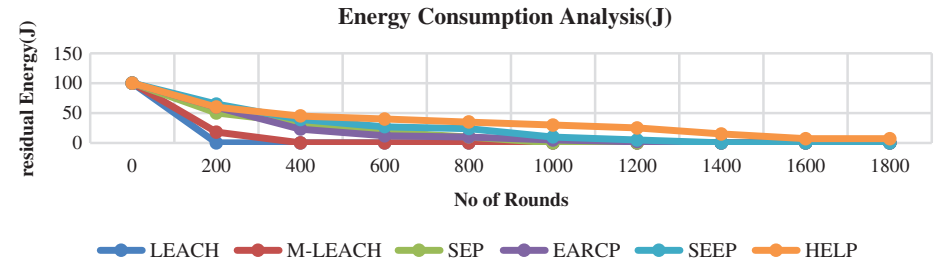

Figure 8: Energy consumption analysis for the different protocols at area of dimension $120 \times 240$ Sq. meters (28800 Sq.meters)

Table 3: Comparative analysis of E2E delay for different algorithms with different zonal area

\begin{tabular}{|c|c|c|c|c|c|}
\hline \multirow{2}{*}{$\frac{\text { Area Dimension }}{60 \times 120 \text { Sq.meters }}$} & \multirow{2}{*}{$\begin{array}{l}\text { Algorithms } \\
\text { LEACH }\end{array}$} & \multicolumn{4}{|c|}{ End to End Delay in (ms) } \\
\hline & & 1.5 & 2 & 2.5 & 3.45 \\
\hline & M-LEACH & 1.34 & 1.9 & 2.4 & 3 \\
\hline & SEP & 1.25 & 1.75 & 1.95 & 2.4 \\
\hline & EARCP & 1.05 & 1.45 & 1.78 & 2 \\
\hline & SEEP & 1.04 & 1.35 & 1.5 & 1.8 \\
\hline & HELP & 0.67 & 0.98 & 1.23 & 1.5 \\
\hline \multirow[t]{6}{*}{$80 \times 160$ Sq.meters. } & LEACH & 3 & 4 & 5 & 6.9 \\
\hline & M-LEACH & 2.6 & 4 & 4.9 & 6 \\
\hline & SEP & 2.5 & 3.89 & 4.7 & 4.8 \\
\hline & EARCP & 2 & 2.9 & 3.6 & 4 \\
\hline & SEEP & 2.5 & 3 & 4 & 4 \\
\hline & HELP & 1.25 & 1.8 & 2.3 & 2.9 \\
\hline \multirow[t]{6}{*}{$100 \times 120$ Sq. meters. } & LEACH & 6 & 8.5 & 11 & 15 \\
\hline & M-LEACH & 5.9 & 12 & 13.4 & 14 \\
\hline & SEP & 5 & 10 & 10 & 12 \\
\hline & EARCP & 4.2 & 4.9 & 8 & 9.2 \\
\hline & SEEP & 5 & 6 & 8 & 8 \\
\hline & HELP & 1.25 & 1.8 & 3 & 3.5 \\
\hline \multirow[t]{6}{*}{$120 \times 240$ Sq. meters. } & LEACH & 9 & 13 & 17 & 28 \\
\hline & M-LEACH & 11 & 19 & 29 & 35 \\
\hline & SEP & 10 & 14 & 20 & 27 \\
\hline & EARCP & 8 & 9.2 & 12 & 19 \\
\hline & SEEP & 7 & 9 & 11 & 16 \\
\hline & HELP & 4 & 6 & 8 & 9 \\
\hline
\end{tabular}




\subsubsection{E2e (End to End) Delay Analysis}

To evaluate the E2E delay analysis, we have deployed the number of 100 nodes at different area dimensions as in the previous experimentations. Since the proposed HELP architecture has incubated the powerful optimization algorithm for the different zonal areas, E2E delay (latency) has very much reduced for effective data transmission,

From Tab. 3, the adoption of optimal algorithms in the proposed framework has reduced the delay in transmission even though the area of implementation has increased. It is noticed that both SEEP and HELP exhibits the same characteristics at less area of coverage while the SEEP framework has reduced performance when the area increases. As the area increases, the effective delay transmission of the proposed algorithm is $16.66 \%$ lesser than SEEP $33.3 \%$ than EARCP, $50 \%$ SEP, M-LEACH and $60 \%$ than LEACH respectively. Since the optimal path is chosen for data transmission, the end to end delay than the other existing algorithms and finds it suitability for prolonged network lifetime in WSN -assisted IoT Networks.

\section{Conclusion}

The WSN assisted IoT devices are equipped with batteries and hence the energy consumption plays an important role in the increased lifetime of the network. In this research, a novel energyefficient routing algorithm based on the integration of machine learning and hybrid optimization along with the multi-tier architecture has been proposed. The paper introduces the new approach of implementing the learning models over the base station for an accurate selection of $\mathrm{CHs}$, which depends on the energy and distance. The performance of Extreme Learning machines in terms of accurate detection of cluster heads has been analyzed and compared with the other existing algorithms. Which in turn proves its suitability for the process of selection when compared with the other traditional algorithms. Furthermore, the integration of hybrid optimization to determine the energy-efficient routing protocol is scalable to larger networks. Extensive experiments were conducted and various metrics were analyzed.

It is found that the proposed HELP for static networks has a better network lifetime by $6.05 \%$ than SEEP, $20.4 \%$ than EARCP, 33\% than SEP 70\%, than LEACH and M-LEACH and consumes less energy by $11 \%$ than SEEP, $25 \%$ than EARCP, 34\% than SEP, $75 \%$ than LEACH and M-LEACH. Moreover, the proposed framework has exhibited better performance in terms of high throughput and less delay during the data transmission. However, the performance of the proposed algorithm can be enhanced by the integration of deep reinforcement learning methods and adaptive metaheuristic optimized routing techniques.

Funding Statement: The authors received no specific funding for this study.

Conflicts of Interest: The authors declare that they have no conflicts of interest to report regarding the present study.

\section{References}

[1] Q. Jing, A. V. Vasilakos, J. Wan, J. Lu and D. Qiu, "Security of the internet of things: Perspectives and challenges," Wireless Networks, vol. 20, no. 8, pp. 2481-2501, 2014.

[2] C. W. Tsai, C. F. Lai and A. V. Vasilakos, "Future internet of things: Open issues and challenges," Wireless Networks, vol. 20, no. 8, pp. 2201-2217, 2014.

[3] K. Jaiswal and V. Anand, "EOMR: An energy-efficient optimal multi-path routing protocol to improve qos in wireless sensor network for IoT applications," Wireless Personal Communications, vol. 114, no. 4, pp. 2493-2515, 2021. 
[4] D. W. Sambo, B. O. Yenke, A. Förster and P. Dayang, "Optimized clustering algorithms for large wireless sensor networks: A review," Sensors, vol. 19, pp. 1-27, 2019.

[5] L. Zhao, S. Qu and Y. Yi. "A modified cluster-head selection algorithm in wireless sensor networks based on LEACH," EURASIP Journal on Wireless Communications and Networking, vol. 2018, pp. 1-8, 2018.

[6] P. Sharma, V. K. Jain and A. K. Uprawal, "EMAEER: Enhanced mobility aware energy efficient routing protocol for internet of things," in Proc. Information and Communication Technology (CICT'18), Jabalpur, India, pp. 1-6, 2018.

[7] K. Neetesh and D. P. Vidyarthi, "A green routing algorithm for IoT enabled software defined wireless sensor network," IEEE Sensors, vol. 18, no. 22, pp. 9449-60, 2015.

[8] H. K. Dwi Widode, A. Kurniawan and M. Sigit Arifianto, "Improving topology of LEACH cluster using reinforcement learning method," in Proc. Int. Conf. on Sensors and Nanotechnology, Penang, Malaysia, pp. 1-4, 2019.

[9] A. Chriki, H. Touati and T. Snoussi, "SVM-Based indoor localization in wireless sensor networks," in Proc. 13th Int. Wireless Communications and Mobile Computing Conf. (IWCMC), Valencia, Spain, pp. 1144-1149, 2017.

[10] T. P. Behera, S. K. Mohapatra and U. S. Samal, "I-SEP: An improved routing protocol for heterogeneous wsn for iot based environmental monitoring," IEEE Internet of Things, vol. 7, no. 1, pp. 710-717, 2019.

[11] C. Li, Y. Cai, Y. Yue, C. Shaotang and B. Hang, "A novel data fusion strategy based on extreme learning machine optimized by bat algorithm for mobile heterogeneous wireless sensor networks," IEEE Access, vol. 8, pp. 16067-16072, 2019.

[12] S. Anurag and S. Tripathi, "A multi-tier based clustering framework for scalable and energy efficient WSN-assisted IoT network," Wireless Networks, vol. 26, no. 5, pp. 3471-3493, 2020.

[13] W. Heinzelman, A. Chandrakasan and H. Balakrishnan, "An application-specific protocol architecture for wireless microsensor networks," IEEE Transaction Wireless Communication System, vol. 1, pp. 660670, 2002.

[14] N. M. Abdul, C. C. Tsimenidis and B. S. Sharif, "Energy-aware clustering for wireless sensor networks using particle swarm optimization," in Proc. IEEE 18th Int. Symp. on Personal, Indoor and Mobile Radio Communications, Athens, Greece, pp. 1-5, 2007.

[15] N. Mittal, U. Singh, R. Salgotra and B. S. Sohi, "A boolean spider monkey optimization based energy efficient clustering approach for WSNs," Wireless Network, vol. 24, pp. 2093-2109, 2018.

[16] G. B. Huang, Q. Y. Zhu and C. K. Siew, "Extreme learning machine: Theory and applications," Neurocomputing, vol. 70, no. 1, pp. 489-501, 2006.

[17] X. S. Yang, "Firefly algorithms for multimodal optimization," in Proc. 5th Symp. on Stochastic Algorithms, Foundations and Applications, in Lecture Notes in Computer Science, O. Watanabe and T. Zeugmann (Eds.), vol. 5792, pp. 169-178, 2009.

[18] A. Mukherjee, C. Niloy and B. K. Das, "W hale Optimization Algorithm: an Implementation to Design low-Pass FIR Filter, in Proc. Innovations in Power and Advanced Computing Technologies (i-PACT), pp. $1-5,2017$.

[19] A. Mehmood, Z. Lv, J. Lloret and M. Muneer Umar, "ELDC: An artificial neural network based energy-efficient and robust routing scheme for pollution monitoring in WSNs," IEEE Transactions on Emerging Topics in Computing, vol. 8, no. 1, pp. 106-114, 2020.

[20] S. Pandey, K. Dubey and S. Sharma, "Performance improvement of LEACH protocol using naïve Bayes classifier in wireless sensor networks," in Proc. Int. Conf. on Computing, Communication, and Intelligent Systems (ICCCIS), Greater Noida, India, pp. 263-267, 2021.

[21] S. Yang, Y. Guo, X. Liu, D. Niu and B. Sun, "A localization algorithm based on compressive sensing by K-nearest neighbor classification," in Proc. 13th Int. Conf. on Signal Processing (ICSP), Chengdu, China, pp. 863-867, 2016. 
[22] T. N. Nguyen, V. V. Le, S. I. Chu, B. H. Liu and Y. C. Hsu, "Secure localization algorithms against localization attacks in wireless sensor networks," Wireless Personal Communications, pp. 1-26, 2021. https://doi.org/10.1007/s11277-021-08404-4.

[23] D. T. Do, M. S. Van Nguyen, T. N. Nguyen, X. Li and K. Choi, "Enabling multiple power beacons for uplink of noma-enabled mobile edge computing in wirelessly powered IoT," IEEE Access, vol. 8, pp. 148892-148905, 2020. 